Pacific Journal of Mathematics

THE FOURIER-STIELTJES ALGEBRA OF A TOPOLOGICAL 


\title{
THE FOURIER STIELTJES ALGEBRA OF A TOPOLOGICAL SEMIGROUP WITH INVOLUTION
}

\author{
ANTHONY TO-MING LAU
}

Let $S$ be a topological semigroup with a continuous involution. We study a subalgebra $F(S)$ of the algebra of continuous weakly almost periodic functions on $S . F(S)$ is translation invariant, closed under conjugation and contains constants. When $S$ has an identity, then $F(S)$ is the linear span of the cone of continuous positive definite functions on $S$. We show that there exists a norm $\|\cdot\|_{\Omega}$ on $F(S)$ such that $\left(F(S),\|\cdot\|_{\Omega}\right)$ is a commutative Banach algebra which can be identified with the predual of a $W^{*}$-algebra $W^{*}(S)$. When $S$ is a locally compact group, then $F(S)$ is precisely the Fourier Stieltjes algebra of $S$. We also show that $\sigma(F(S))$, the spectrum of $F(S)$, is a *-semigroup in $W^{*}(S)$, and study the relation of $\sigma\left(F\left(S_{1}\right)\right)$ and $\sigma\left(F\left(S_{2}\right)\right)$ when $F\left(S_{1}\right)$ and $F\left(S_{2}\right)$ are isometric isomorphic Banach algebras.

1. Introduction. Recently, Dunkl and Ramirez [5] defined a subalgebra $R(S)$ of the algebra $W A P(S)$ of complex-valued continuous weakly almost periodic functions on $S$. The algebra $R(S)$, called the representation algebra of $S$, is constructed by considering continuous representations of $S$ into the unit ball of $L_{\infty}(X, \mu)$ with the weak*topology, where $(X, \mu)$ is some probability measure space. They showed that $R(S)$ is translation invariant, closed under conjugation and contains all bounded continuous semi-characters on $S$. Furthermore $R(S)$, with an appropriate norm, becomes a commutative Banach algebra and the dual of $R(S)$ can be identified with a weak*-closed subalgebra of a commutative $W^{*}$-algebra. If $G$ is a commutative locally compact group, then $R(G)=M(\widehat{G})^{\wedge}$, the Fourier Stieltjes transform of the measure algebra on the dual group $\hat{G}$ (see $[6, \mathrm{p}$. 80]).

Our present work deals with the study of the subalgebra $F(S)$ of $W A P(S)$ of a topological *-semigroup $S$ (i.e., a topological semigroup with a continuous involution). If $S$ has an identity, then $F(S)$ is the linear span of continuous positive definite function on $S$. Also if $S$ is a commutative, then $F(S)$ is contained in the representation algebra $R(S)$. We show that $F(S)$ can be identified with the predual of a $W^{*}$-algebra, $W^{*}(S)$. Furthermore $F(S)$ with the predual norm is a commutative Banach algebra, called the Fourier Stieltjes algebra of $S$. The algebra $F(S)$ is also translation invariant, closed under conjugation and contains all continuous ${ }^{*}$-semi-characters of 
S. Also there exists an ultra-weakly continuous *-representation of $S$ into the unit ball of $W^{*}(S)$ "containing" all other ultra-weakly continuous *-representations of $S$ into the unit ball of a $W^{*}$-algebra. In particular, if $G$ is a locally compact group (with involution $g \rightarrow g^{-1}$ ), then $W^{*}(G)$ is the big group algebra defined by John Ernest [9] (see also [8]). Furthermore, if $S$ is commutative and has an identity, then $F(S)$ is isometric and algebra isomorphic to a weak*dense subalgebra of the measure algebra of a compact topological commutative semigroup.

This paper is organized in the following way: In $\S 2$ we list some notations and preliminary properties of topological *-semigroups $S$; definitions and properties of $F(S)$ and $W^{*}(S)$ as stated in the previous paragraph will be made precise in $\S 3$ and 4 . Analysis of the spectrum $\sigma(F(S))$ of $F(S)$ is taken up in $\S 5$. We show that $\sigma(F(S))$ is a *-semigroup in $W^{*}(S)$ and study the relation of $\sigma\left(F\left(S_{1}\right)\right)$ and $\sigma\left(F\left(S_{2}\right)\right)$ when $S_{1}, S_{2}$ are topological *-semigroups, and $F\left(S_{1}\right)$ and $F\left(S_{2}\right)$ are isometric isomorphic Banach algebras.

Continuous positive definite functions on topological *-semigroups $S$ have been studied by R. J. Lindahl and P. H. Maserick [15], and more recently by C. Berg and J. Christensen [3] for commutative $S$ with involution on $S$ given by the identity map. Our analysis of the spectrum of $F(S)$ is inspired and motivated by the work of Martin E. Walter in [18] and [19].

It is our pleasure to thank the referee of this paper. His many valuable suggestions have much improved the contents of the original version of our work.

2. Preliminaries and some notations. Let $A$ be a subset of a linear space $E$, then $\langle A\rangle$ will denote the linear span of $A$. If $E$ is also a normed linear space, then the closure of $A$ and the closed linear span of $A$ will be denoted by $\bar{A}$ and $\langle A\rangle^{-}$respectively if the closure is taken with respect to the norm topology, or by $\bar{A}^{\tau}$ and $\langle A\rangle^{-\tau}$ respectively if the closure is taken with respect to a topology $\tau$ on $E$ different from the norm topology.

The continuous dual of a normed linear space $E$ will be denoted by $E^{*}$. If $x \in E$ and $\phi \in E^{*}$, then the value of $\phi$ at $x$ will be denoted by $\phi(x)$ or $\langle\phi, x\rangle$. Also if $F \subseteq E^{*}$, then $\sigma(E, F)$ will denote the locally convex topology on $E$ determined by the semi-norms $\left\{p_{\phi} ; \phi \in F\right\}$, where $p_{\phi}(x)=|\phi(x)|$ for all $x \in E$.

If $M$ is a $W^{*}$-algebra, then $M_{*}$ will denote its unique predual. For each $x \in M$, and $\phi \in M_{*}$, write $L_{x} \phi, R_{x} \phi$ and $\phi^{*}$ as the functionals in $M_{*}$ defined by $L_{x} \phi(y)=\phi(x y), R_{x} \phi(y)=\phi(y x)$ and $\phi^{*}(y)=\overline{\phi\left(y^{*}\right)}$ for each $y \in M$. Also the ultraweak topology on $M$ (i.e., the $\sigma\left(M, M_{*}\right.$ )topology) will often be written as the $\sigma$-topology. 
By a topological semigroup $S$, we shall mean a semigroup $S$ with a Hausdorff topology such that for each $a \in S$, the mappings $s \rightarrow a s$ and $s \rightarrow s a$ from $S$ into $S$ are continuous.

Let $S$ be a topological semigroup and let $C(S)$ be the space of bounded continuous complex-valued functions on $S$. For each $a \in S$, define the left and right translation operators $\iota_{a}, r_{a}$ on $C(S)$ by:

$$
\begin{aligned}
& \left(\ell_{a} f\right)(s)=f(a s) \\
& \left(r_{a} f\right)(s)=f(s a)
\end{aligned}
$$

for each $s \in S$. A function $f \in C(S)$ is weakly almost periodic if $\left\{\ell_{a} f ; a \in S\right\}$ is relatively compact in the weak topology of $C(S)$. Then, as known, the space $W A P(S)$ of continuous weakly almost periodic functions on $S$ is a translation invariant closed subalgebra of $C(S)$ containing constants.

By an involution on a topological semigroup $S$ we shall mean a map from $S$ into $S$, denoted by $s \rightarrow s^{*}$, such that

$$
\begin{gathered}
(a b)^{*}=b^{*} a^{*} \\
a^{* *}=a
\end{gathered}
$$

for all $a \in S$. A topological *-semigroup is a topological semigroup with a fixd continuous involution.

REMARK 2.1. (a) Not all topological semigroups admit an involution (see $[15, \mathrm{p} .771]$ ).

(b) If $S$ is commutative, then the identity map on $S$ defines an involution on $S$.

(c) If $S$ has an identity $u$, then $u^{*}=u$.

(d) If $M$ is a $W^{*}$-algebra, then the unit ball of $M$ with the $\sigma$-topology is a compact topological ${ }^{*}$-semigroup with the multiplication and involution of $M$.

If $S$ is a topological *-semigroup, $f \in C(S)$, define $f^{*} \in C(S)$ by $f^{*}(s)=\overline{f\left(s^{*}\right)}$ for all $s \in S$. Then the map $f \rightarrow f^{*}$ defines an involution on the Banach algebra $C(S)$.

A complex-valued function $f$ on a topological *-semigroup $S$ is called positive definite if for any complex numbers $\lambda_{1}, \cdots, \lambda_{n}$ and any $s_{1}, \cdots, s_{n}$ in $S$, we have

$$
\sum_{i=1}^{n} \sum_{j=1}^{n} \bar{\lambda}_{i} \lambda_{j} f\left(s_{i}^{*} s_{j}\right) \geqq 0 \text {. }
$$

The collection of continuous positive definite functions on $S$ will be denoted by $P(S)$. The next proposition can be proved by an argument similar to that in [12, 32.9] (see also [15, Theorem 3.4]). 
Proposition 2.2. Let $S$ be a topological *-semigroup. Then $P(S)$ is a cone in $C(S)$ closed under conjugation, involution and pointwise product.

When $G$ is a group, then the involution on $G$, unless otherwise specified, will be the one defined by the inversion map $g \rightarrow g^{-1}, g \in G$.

3. The Fourier Stieltjes algebra $F(S)$. Throughout this section, $S$ will denote a topological *-semigroup.

By a representation of $S$ we shall mean a pair $(\omega, M)$, where $M$ is a $W^{*}$-algebra and $\omega$ is a homomorphism of $S$ into $M_{1}=$ $\{x \in M ;\|x\| \leqq 1\}$ regarded as a semigroup with multiplication from $M$ i.e., $\omega(a b)=\omega(a) \omega(b)$ for all $a, b \in S$. The representation $(\omega, M)$ is a *-representation if $\omega\left(a^{*}\right)=\omega(a)^{*}$ for all $a \in S$; it is $\sigma$-continuous if $\omega$ is continuous when $M_{1}$ has the $\sigma$-topology.

REMARK 3.1. If $S$ has an identity $u$, and $(\omega, M)$ is a *-representation of $S$, then $\omega(u)=p$ is a projection in $M$, and $\omega(S)$ is contained in the $W^{*}$-algebra $p M p$ for which $p$ is the identity. Also if $\langle\omega(S)\rangle^{-\sigma}=M$, then $\omega(u)$ is the identity of $M$.

If $(\omega, M)$ is a $\sigma$-continuous *-representation of $S$ such that $\langle\omega(S)\rangle$ is $\sigma$-dense in $M$, then $\operatorname{card}\left(M_{*}\right) \leqq c^{\operatorname{car}(S)}$, where $c$ is the cardinality of the real numbers. Hence we may form the collection $\Omega(S)$ of all $\sigma$-continuous *-representations $\alpha=(\omega, M)$ of $S$ such that $\langle\omega(S)\rangle^{\sigma}=M$. Let $F(S)$ denote all complex-valued functions $f$ such that $f=\hat{\psi}$ for some $\omega \in M_{*}$ and some $\alpha=(\omega, M)$ in $\Omega(S)$. For each $f \in F(S)$, let

$$
\begin{aligned}
& \|f\|_{\infty}=\sup \{|f(s)| ; s \in S\} \\
& \|f\|_{\Omega}=\inf \left\{\|\psi\| ; \psi \in M_{*}, \hat{\psi}=f \text { and }(\omega, M) \in \Omega(S)\right\}
\end{aligned}
$$

THEOREM 3.2. (a) $F(S)$ is a subalgebra of $W A P(S)$ containing the constant functions. Furthermore, $\|\cdot\|_{\Omega}$ is a norm on $F(S)$ and $\left(F(S),\|\cdot\|_{\Omega}\right)$ is a commutative normed algebra with unit.

(b) If $f \in F(S)$ and $a \in S$, then the functions $r_{a} f, \iota_{a} f, f^{*}, \bar{f}$ are all in $F(S)$ and $\left\|r_{a} f\right\|_{\Omega} \leqq\|f\|_{\Omega},\left\|\epsilon_{a} f\right\|_{\Omega} \leqq\|f\|_{\Omega},\left\|f^{*}\right\|_{\Omega}=\|f\|_{\Omega},\|\bar{f}\|_{\Omega}=$ $\|f\|_{\Omega}$ and $\|f\|_{\infty} \leqq\|f\|_{\Omega}$.

Proof. That $F(S) \subseteq W A P(S)$ follows from [14, Lemma 6.3]. The remainder of the theorem can be proved quite similarly to [6, Theorem 2.1.6], we omit the details.

We shall call $\left(F(S),\|\cdot\|_{\Omega}\right)$ the Fourier Stieltjes algebra of $S$.

REMARK 3.3. (a) The algebra $F(S)$ cannot be enlarged and the 
norm on $F(S)$ cannot be decreased by considering a collection $\mathscr{C}$ of $\sigma$-continuous *-representations of $S$ containing $\Omega(S)$. Indeed, if $f \in F(S)$ and $\alpha=(\omega, M) \in \mathscr{C}$ such that $f=\hat{\psi}$ for some $\psi \in M_{*}$, let $N=\langle\omega(S)\rangle^{-\sigma}$ and $\psi_{0}$ be the restriction of $\psi$ to $N$. Then $(\omega, N) \epsilon$ $\Omega(S), \hat{\psi}_{0}=f$ and $\left\|\psi_{0}\right\| \leqq\|\psi\|$.

(b) If $S$ is commutative, then $F(S) \subseteq R(S)$, where $R(S)$ is the representation algebra of $S$ defined by Dunkl and Ramirez [5]. To see this, let $f \in F(S)$. Choose $(\omega, M) \in \Omega(S)$ such that $\hat{\phi}=f$ for some $\phi \in M_{*}$. Let $X$ be the spectrum of $M$. Then $\operatorname{card}(X) \leqq$ $c^{\mathrm{card}(S)}$. By the Riesz representation theorem, there exists a probability measure $\mu_{\phi}$ on $X$ such that $\dot{\phi}(a)=\int_{X} a(t) d \mu_{\phi}(t)$ for each $a \in M$. Consider the mapping $\Phi_{\phi}$ from $M$ into $L_{\infty}\left(X, \mu_{\phi}\right)$ defined by $\Phi_{\phi}(a)=\hat{a}$, where $\hat{a}$ is the Gelfand transform of $a$. Then $\Phi_{\phi}$ is a $W^{*}$-homomorphism of $M$ into $L_{\infty}\left(X, \mu_{\phi}\right)$ (see [16, p. 46]). Define a presentation $\left(\omega_{\phi}, L_{\infty}\left(X, \mu_{\phi}\right)\right)$,of $S$ by $\omega_{\phi}(S)=\Phi_{\phi}(\omega(s))$. Then $\hat{\phi}(s)=\left\langle 1, \omega_{\phi}(s)\right\rangle$ for all $s \in S$. Hence $f=\hat{\phi} \in R(S)$.

Note that the inclusion $F(S) \subseteq R(S)$ may be proper (see Example 4.2).

(c) If $S$ is an idempotent commutative topological semigroup with involution $s^{*}=s$ for all $s \in S$, then any representation $(\omega, M)$ of $S$, where $M$ is a commutative $W^{*}$-algebra, is a *-representation. In particular $F(S)=R(S)$. Indeed, we may assume that $M=L_{\infty}(X, \mu)$ for some measure space $(X, \mu)$. Since $\omega(s)^{2}=\omega\left(s^{2}\right)=\omega(s)$ for all $s \in S$, it follows that $\omega(s)$ is a characteristic function on some subset of $\Omega$. Hence $\omega(s)^{*}=\omega\left(s^{*}\right)$.

(d) Let $G$ be an abelian group. Then for any representation $(\mu, M)$ where $M$ is a commutative $W^{*}$-algebra, is a ${ }^{*}$-representation of $G$. Consequently $F(G)=R(G)$. Indeed, write $M=L_{\infty}(X, \mu)$ for some measure space $(X, \mu)$. We may assume that $\omega(u)=1$, where $u$ is the identity of $G$. Then for each $g \in G, \omega(g) \omega\left(g^{-1}\right)=\omega(u)=1$. Hence $|\omega(g)|=1$ and $\omega\left(g^{-1}\right)=\overline{\omega(g)}=\omega(g)^{*}$.

(e) If $S$ is the unit ball of a $W^{*}$-algebra $M$, then the restriction map is a linear isometry from $M_{*}$ into $F(S)$.

(f) A function $\chi: S \rightarrow C$ is called a semi-character if $|\chi(s)| \leqq 1$ and $\chi(s \cdot t)=\chi(s) \chi(t)$ for all $s, t \in S$. A continuous semi-character $\chi$ is in $F(S)$ if and only if $\chi\left(s^{*}\right)=\overline{\chi(s)}$ for all $s \in S$. In this case $\chi \in P(S)$ and $\|\chi\|_{\Omega}=1$ whenever $\chi$ is nonzero, (see [6, Remark 2.1.8]).

The next proposition follows easily from [15, Theorem 3.2] and Remark 3.3(a):

Proposition 3.4. If $S$ has an identity, then $F(S)=\langle P(S)\rangle$.

REMARK 3.5. (a) Let $S_{u}$ denote the semigroup formed by ad- 
joining to $S$ an identity $u$. Equip $S_{u}$ with the topology $\eta$ that a subset $0 \subseteq S_{u}$ is in $\eta$ if and only if $0 \cap \mathrm{S}$ is open in $S$. Then $\left(S_{u}, \eta\right)$ is a topological semigroup. Also the involution on $S$ can be extended to an involution on $S_{u}$ by defining $u^{*}=u$. Let $r$ denote the restriction map from $F\left(S_{u}\right)$ into $F(S)$. Then $r$ is norm decreasing, onto and $r\left(P\left(S_{u}\right)\right)=F(S) \cap P(S)$.

(b) The assumption that $S$ has an identity cannot be removed from Proposition 3.4. Indeed, let $S$ be a set with at least two elements. Let $z \in S$ be fixd. Define on $S$ the multiplication $a b=z$ for all $a, b \in S$. Equip $S$ with the discrete topology and involution $a=a^{*}$ for all $a \in S$. Pick $w \in S, w \neq z$. Let $f$ be the characteristic function on the set $\{w\}$. Then $f \in P(S)$, but $f \notin F(S)$. Indeed, there exists no $k$ such that

$$
\left|\sum_{i=1}^{n} c_{i} f\left(s_{i}\right)\right|^{2} \leqq k \sum_{i, j=1}^{n} c_{i} \bar{c}_{j} f\left(s_{i} s_{j}^{*}\right)
$$

for any $s_{1}, \cdots, s_{n}$ in $S$ and complex numbers $c_{1}, \cdots, c_{n}$. Hence by Corollary 1.2 in [15], $f$ is not extendable to a function in $P\left(S_{u}\right)$. By (a), $f \in F(S)$.

4. The operator algebra $W^{*}(S)$. Let $S$ be $a$ topological *-semigroup and write $M_{\Omega}=\sum \oplus M_{\alpha}$, the direct summand of the $W^{*}$ algebras $M_{\alpha}, \alpha \in \Omega(S)$. (See [16, p. 2].) Define a *-homomorphism of $S$ into $M_{\Omega}$ by: $\omega_{\Omega}(s)(\alpha)=\omega_{\alpha}(s)$ for each $\alpha=\left(\omega_{\alpha}, M_{\alpha}\right)$ in $\Omega(S)$. Then

$$
\left\|\omega_{\Omega}(s)\right\|=\sup \left\{\left\|\omega_{\alpha}(s)\right\| ; \alpha \in \Omega(S)\right\} \leqq 1
$$

for each $s \in S$. Also if $s_{n}$ is a net in $S$ converging to some $s \in S$, then the net $\left\langle\omega_{\Omega}\left(s_{n}\right)(\alpha), \psi\right\rangle=\left\langle\omega_{\alpha}\left(s_{n}\right), \psi\right\rangle$ converges to $\left\langle\omega_{\Omega}(s)(\alpha), \psi\right\rangle$ for each $\alpha \in \Omega(S)$ and $\psi \in\left(M_{\alpha}\right)_{*}$. Since the $\sigma$-topology on $M_{\Omega}$ agrees with the topology determined by the semi-norms $\left\{P_{\alpha, \psi} ; \alpha \in \Omega(S)\right.$, $\left.\psi \in\left(M_{\alpha}\right)_{*}\right\}$ on the unit ball, where

$$
\left|P_{\alpha, \psi}(x)\right|=|\langle x(a), \psi\rangle|
$$

for each $x \in M_{\Omega}$, it follows that $\left(\omega_{\Omega}, M_{\Omega}\right)$ is a $\sigma$-continuous *-representation of $S$. Write

$$
W^{*}(S)=\left\langle\omega_{\Omega}(S)\right\rangle^{-\sigma}
$$

THEOREM 4.1. Let $S$ be a topological *-semigroup. Then:

(a) The mapping $\pi: W^{*}(S)_{*} \rightarrow F(S)$ defined by $\pi(\psi)=\hat{\psi}, \psi \epsilon$ $W^{*}(S)_{*}$, is a linear isometry from $W^{*}(S)_{*}$ onto $F(S)$. Consequently, the normed algebra $F(S)$ is complete. Furthermore, $\pi(\psi)$ is positive definite if and only if $\psi$ is positive. 
(b) If $(\omega, M)$ is any $\sigma$-continuous *-representation of $S$, then there exists a $W^{*}$-homomorphism $h_{\omega}$ from $W^{*}(S)$ into $M$ such that the diagram

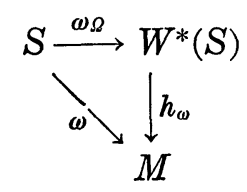

is commutative. Also if $\psi \in M_{*}$, then $\langle x, \hat{\psi}\rangle=\left\langle h_{\omega}(x), \psi\right\rangle$ for all $x \in W^{*}(S)$.

Proof of Theorem 4.1 is rather routine. We omit the details.

EXAMPLE 4.2. Let $\boldsymbol{Z}$ be the group of integers with addition and involution $n \rightarrow-n$. Then $F(\boldsymbol{Z})=R(\boldsymbol{Z})=\langle P(\boldsymbol{Z})\rangle$ (Remark 3.3(d) and Proposition 3.4), and $W^{*}(\boldsymbol{Z})$ is the commutative $W^{*}$-algebra $C(T)^{* *}$, where $T$ is circle group (see Remark 4.3(b)).

On the other hand, if $\boldsymbol{Z}$ has involution $n \rightarrow n$, then $F(\boldsymbol{Z})=C^{2}$, $W^{*}(\boldsymbol{Z})=C^{2}$ and hence $F(\boldsymbol{Z})$ is a proper subset of $R(\boldsymbol{Z})$ (see Remark $3.3(\mathrm{a}))$. To see this, consider any $(\omega, M) \in \Omega(Z)$. Then $M$ is a commutative $W^{*}$-algebra. Hence $\omega(n)=\overline{\omega(n)}=\omega(-n)$ by Remark 3.3 (d). Consequently $\omega(n)^{2}=\omega(0)=1$ for all $n \in Z$ and $\omega(Z)$ has at most two elements. However, if $M$ is the subalgebra of $L_{\infty}[0,1]$ generated by the functions $1, h$, where

$$
h(t)=\left\{\begin{aligned}
1 & \text { if } \quad 0 \leqq t<\frac{1}{2} \\
-1 & \text { if } \quad \frac{1}{2} \leqq t \leqq 1
\end{aligned}\right.
$$

then $\omega(0)=1$ and $\omega(1)=\omega(-1)=h$ defines a representation of $Z$ in $\Omega(\boldsymbol{Z})$, and $\langle\omega(\boldsymbol{Z})\rangle=M$, which is two-dimensional. Hence $W^{*}(\boldsymbol{Z})=C^{2}$ and $F(Z)=C^{2}$.

REMARK 4.3. (a) Let $S$ be a locally compact topological *-semigroup. Let $M(S)$ be the Banach algebra of complex, finite, regular Borel measures on $S$ with multiplication of two elements $\mu, \nu$ in $M(S)$ defined by

$$
\int f d \mu * \nu=\iint f(s t) d \mu(s) d \nu(t)
$$

for all $f \in C_{0}(S)$, and total variation norm, where $C_{0}(S)$ is the space of all functions $f \in C(S)$ vanishing at infinity (see [11]). For each $\mu \in M(S)$, define $\mu^{*} \in M(S)$ to be the measure representing the func- 
tional $f \rightarrow \overline{\sqrt{f^{*}(t) d \mu(t)}}$ on $C_{0}(S)$. Then as observed in [14, Lemma 6.8], the $\operatorname{map} \mu \rightarrow \mu^{*}$ is an involution on $M(S)$ with $\|\mu\|=\left\|\mu^{*}\right\|$ and $\left(\varepsilon_{a}\right)^{*}=\varepsilon_{a^{*}}$ for each $a \in S$, where $\varepsilon_{a}$ is the point measure at $a$.

Define

$$
\left\langle\tilde{\boldsymbol{\omega}}_{\Omega}(\mu), \phi\right\rangle=\int \hat{\phi}(t) d \mu(t)
$$

for each $\mu \in M(S)$, and $\phi \in W^{*}(S)_{*}$. Clearly $\tilde{\omega}_{\Omega}\left(\varepsilon_{a}\right)=\omega(\alpha)$ for each $a \in S$. Consequently $\tilde{\omega}_{\Omega}$ defines a norm-decreasing *-homomorphism of $M(S)$ into $W^{*}(S)$ which is continuous when $M(S)$ has the $\sigma(M(S), F(S))$ topology and $W^{*}(S)$ has the $\sigma$-topology. Furthermore, if $\beta$ is any norm-decreasing *-homomorphism from $M(S)$ into a $W^{*}$ algebra $M$ which is continuous when $M(S)$ has the $\sigma(M(S), F(S))$ topology and $M$ has the $\sigma$-topology, then there exists a $W^{*}$-homomorphism $h$ from $W^{*}(S)$ into $M$ such that the diagram

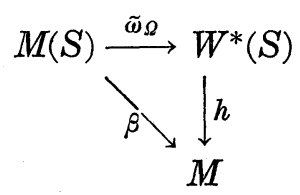

is commutative.

(b) Let $G$ be a locally compact group. Let $C^{*}(G)$ denote the completion of $L_{1}(G)$ with the norm

$$
\|h\|_{c}=\sup \left\{\left\|T_{h}\right\|\right\}, \quad h \in L_{1}(G),
$$

where the suprenum is taken over all no-where trivial *-representation $T$ of $L_{1}(G)$ as an algebra of bounded linear operators on a Hilbert space. Then as well known (see [10, Chapter 2]), $\langle P(G)\rangle$ can be identified with the dual of $C^{*}(G)$, and $P(G)$ is precisely the positive linear functionals on the $C^{*}$-algebra $C^{*}(G)$. In this case $F(G)=\langle P(G)\rangle$ (see Proposition 3.4) and $\|f\|_{\Omega}$ is precisely the norm of $f$ regarded as a linear functional on $C^{*}(G)$ for each $f \in F(G)$. Furthermore, $W^{*}(G)$ is isomorphic to the second conjugate algebra of $C^{*}(G)$ with the Arens product. (See [8, Remark 2.6 and Proposition 2.8].)

(c) Let $S$ be a topological *-semigroup and let $C_{\Omega}^{*}(S)=\left\langle\omega_{\Omega}(S)\right\rangle^{-}$. Then $C^{*}(S)$ is a $C^{*}$-subalgebra of $W^{*}(S)$. For each $f \in F(S), x \in C_{\Omega}^{*}(S)$, define $f \cdot x \in W^{*}(S)$ by

$$
\langle f \cdot x, g\rangle=\langle x, f \cdot g\rangle
$$

for all $g \in F(S)$. Then $f \cdot x \in C_{\Omega}^{*}(S)$. Also if $m \in C_{*}^{*}(S)^{*}$ and $x \in C_{\Omega}^{*}(S)$, then the element $m_{L}(x)$ in $W^{*}(S)$ defined by 


$$
\left\langle m_{L}(x), f\right\rangle=\langle m, f \cdot x\rangle
$$

for all $f \in F(S)$ is also in $C_{\Omega}^{*}(S)$. Hence we may define on $C_{\Omega}^{*}(S .)^{*}$ the Arens product [1] by

$$
\langle n \cdot m, x\rangle=\left\langle n, m_{L}(x)\right\rangle
$$

for any $n, m \in C_{\Omega}^{*}(S), x \in C_{\Omega}^{*}(S)$. Then $C_{\Omega}^{*}(S)^{*}$ with product and the dual norm is a commutative Banach algebra containing an isometric copy of $F(S)$. Furthermore, multiplication in the unit ball of $C_{\Omega}^{*}(S)^{*}$ is jointly continuous with respect to the weak*-topology. (This follows from our [14, Theorem 6.11] when $S$ has an identity; otherwise just use an argument similar to the proof given there.)

(d) Let $S$ be a topological *-semigroup and let $S_{d}$ denote $S$ with the discrete topology. For each $m \in C_{\Omega}^{*}(S)^{*}$, define $\hat{m}(s)=$ $m\left(\omega_{\Omega}(s)\right)$ for all $s \in S$. Then clearly $\hat{m} \in\left\langle P\left(S_{d}\right)\right\rangle$. Since $\left(\omega_{\Omega}, M\right) \in \Omega(S)$, where $M$ is the enveloping $W^{*}$-algebra of $C_{\Omega}^{*}(S)$, it follows that $\hat{m} \in F\left(S_{d}\right)$ and $\|\hat{m}\|_{\Omega} \leqq\|m\|$. A simple computation shows that the map $\tilde{\pi}: C_{\Omega}^{*}(S)^{*} \rightarrow F\left(S_{d}\right)$ defined by $\tilde{\pi}(m)=\hat{m}, m \in C_{\Omega}^{*}(S)^{*}$, is normdecreasing algebra isomorphism from $C_{\Omega}^{*}(S)^{*}$ into $F\left(S_{d}\right)$. In particular if $S$ is discrete, then $F(S)$ is isometric and algebra isomorphic to $C_{\Omega}^{*}(S)^{*}$.

(e) Let $S$ be a commutative topological *-semigroup with an identity $u$, and let $\widehat{S}_{d}$ (resp. $\widehat{S}$ ) denote the set of all (resp. continuous) nonzero *-semi-characters on $S$ i.e., semi-characters $\chi$ on $S$ such that $\chi\left(s^{*}\right)=\overline{\chi(s)}$ for all $s \in S$. Note that $\chi(u)=1$ for each $\chi \in \widehat{S}_{d}$. Equip $\widehat{S}_{d}$ with the topology of pointwise convergence. Then $\hat{S}_{d}$ with pointwise multiplication is a compact togological semigroup. Let $\Delta(S)$ denote the spectrum of the commutative $C^{*}$-algebra $C_{\Omega}^{*}(S)$ and write $\Delta(S)^{\wedge}=\tilde{\pi}\left(\Delta(S)\right.$ ) where $\tilde{\pi}$ is as defined in (d) above. Then $\Delta(S)^{\wedge}$ is a compact subsemigroup of $\hat{S}_{d}$ containing $\hat{S}$. Furthermore, it follows from [14, Theorem 6.12] that there exists a linear isometry and algebra homomorphism from $F(S)$ into a weak*-dense subalgebra of the measure algebra $M\left(\Delta(S)^{\wedge}\right)$. Also if $S$ is a discrete commutative *-semigroup with an identity, then $F(S)$ is isometric and algebra isomorphic to $M(\hat{S})$.

5. The spectrum of $F(S)$. Throughout this section $S$ will denote a topological *-semigroup and $\sigma(F(S))$ will denote the spectrum of $F(S)$ i.e., the collection of all nonzero multiplicative linear functionals on $F(S)$.

Recently Martin E. Walter [18] [19] has given detailed analysis on the spectrum of the Fourier Stieltjes algebra of a locally compact group. In this section, we shall generalize some of Walter's results to the spectrum of $F(S)$. We begin with the following simple observations. 
Let $x \in W^{*}(S)$ and $f \in F(S)$. Define a bounded complex-valued function $x_{l}(f)$ on $S$ by

$$
x_{l}(f)(s)=\left\langle x, l_{s} f\right\rangle
$$

for each $s \in S$. Let $\phi \in W^{*}(S)_{*}$ such that $\hat{\phi}=f$. Then $\left(L_{\omega_{\Omega}(S)} \dot{\phi}\right)^{\wedge}=$ $l_{s} f$. Hence

$$
\begin{aligned}
x_{l}(f)(s) & =\left\langle x, L_{\omega_{\Omega}(s)} \phi\right\rangle \\
& =\left\langle R_{x} \phi, \omega_{\Omega}(s)\right\rangle \\
& =\left(R_{x} \phi\right)^{\wedge}(s)
\end{aligned}
$$

for all $s \in S$. Consequently $x_{l} f \in F(S)$ and $\left\|x_{l} f\right\|_{\Omega} \leqq\|x\|\|f\|_{\Omega}$. Hence if $y \in W^{*}(S)$, we may define an element $y \circ x$ in $W^{*}(S)$ by

$$
\langle y \circ x, f\rangle=\left\langle y, x_{l}(f)\right\rangle
$$

for all $f \in F(S)$.

Lemma 5.1. If $x, y \in W^{*}(S)$, then $y \circ x=y \cdot x$, where $y \cdot x$ denotes the product of $y, x$ in $W^{*}(S)$. Consequently $(x \cdot y)_{l}(f)=y_{l}\left(x_{l}(f)\right)$ for each $f \in F(S)$.

Proof. Let $x \in W^{*}(S)$ be fixed. The equation $y \circ x=y \cdot x$ clearly holds for all $y=\omega_{\Omega}(s), s \in S$. Hence it holds for all $y \in\left\langle\omega_{\Omega}(S)\right\rangle$. Now if $y \in W^{*}(S)$ and $y_{\alpha}$ is a net in $\left\langle\omega_{\Omega}(S)\right\rangle$ converging to $y$ in the $\sigma$-topology, then for each $f \in F(S)$,

$$
\begin{aligned}
\langle y \circ x, f\rangle & =\left\langle y, x_{l}(f)\right\rangle \\
& =\lim _{\alpha}\left\langle y_{\alpha}, x_{l}(f)\right\rangle \\
& =\lim _{\alpha}\left\langle y_{\alpha} \circ x, f\right\rangle \\
& =\lim _{\alpha}\left\langle y_{\alpha} \cdot x, f\right\rangle \\
& =\langle y \cdot x, f\rangle
\end{aligned}
$$

by $[16$, p. 18]. The final assertions from direct computation.

LEMma 5.2. (a) If $x \in \sigma(F(S))$, then $x_{l}$ is an algebra homomorphism from $F(S)$ into $F(S)$.

(b) If $S$ has an identity, and $x$ is a nonzero element in $W^{*}(S)$ such that $x_{l}$ is an algebra homomorphism, then $x \in \sigma(F(S))$.

Proof. (a) If $f, g \in F(S)$, then

$$
x_{l}(f \cdot g)(s)=\left\langle x, l_{s}(f \cdot g)\right\rangle=\left\langle x,\left(l_{s} f\right)\left(l_{s} g\right)\right\rangle=\left\langle x, l_{s} f\right\rangle\left\langle x, l_{s} g\right\rangle
$$

for all $s \in S$. Hence $x_{l}$ is an algebra homomorphism.

(b) follows simply by evaluation at the identity. 
REMARK 5.3. Note that Lemma $5.2(\mathrm{~b})$ is false when $S$ does not have an identity as the following example shows. Let $S=\left\{s_{1}, s_{2}, s_{3}\right\}$ with multiplication defined by

$$
x_{i} \cdot x_{j}= \begin{cases}x_{i} & \text { if } i=j \\ x_{1} & \text { if } i \neq j\end{cases}
$$

Then $S$ is commutative and has no identity. Let $S$ have the discrete topology and involution defined by the identity map. Then $F(S)$ separate points. In fact, let $M=L_{\infty}[0,1]$ and define a *-representation $\omega$ of $S$ into $M$ by $\omega\left(s_{1}\right)=0, \omega\left(s_{2}\right)=1_{[0,1 / 2)}$, and $\omega\left(s_{3}\right)=1_{[1 / 2,1]}$. Then $F_{\omega}(S)$ clearly separate points and contained in $F(S)$. Hence $\sigma(F(S))=\omega_{\Omega}(S)$ consists of three distinct points, and the identity $e$ of $W^{*}(S)$ is not in $\sigma(F(S))$. However $e_{l}$, being the identity operator on $F(S)$, is an algebra homomorphism.

Proposition 5.4. If $x, y \in \sigma(F(S))$, then $x^{*}$ and $x \cdot y$ are in $\sigma(F(S))$.

Proof. Let $f, g \in P(S) \cap F(S)$. Then $f \cdot g \in P(S) \cap F(S)$ by Proposition 2.2. Hence if $x \in \sigma(F(S))$, then $x^{*} \neq 0$ and

$$
\left\langle x^{*}, f \cdot g\right\rangle=\overline{\langle x, f \cdot g\rangle}=\overline{\langle x, f\rangle\langle x, g\rangle}=\left\langle x^{*}, f\right\rangle\left\langle x^{*}, g\right\rangle
$$

by Theorem 4.1 (b). Since $\langle P(S) \cap F(S)\rangle=F(S)$, it follows that $x^{*} \in \sigma(F(S))$.

If $x, y \in \sigma(F(S))$ and $f, g \in F(S)$, then

$$
\begin{aligned}
\langle x \cdot y, f \cdot g\rangle & =\left\langle x, y_{l}(f \cdot g)\right\rangle=\left\langle x, y_{l}(f) y_{l}(g)\right\rangle=\left\langle x, y_{l}(f)\right\rangle\left\langle x, y_{l}(g)\right\rangle \\
& =\langle x \cdot y, f\rangle\langle x \cdot y, g\rangle
\end{aligned}
$$

using Lemmas 5.1 and 5.2. To see that $x \cdot y \neq 0$, we observe that if 1 is the constant one function on $S$, then $\langle x, 1\rangle=1$. Hence

$$
\langle x \cdot y, 1\rangle=\left\langle x, y_{l}(1)\right\rangle=\langle x, 1\rangle=1
$$

using Lemma 5.1 again. Hence $x \cdot y \in \sigma(F(S))$.

If $\left(\omega_{i}, M\right), i=1,2$, are $\sigma$-continuous *-representations of $S$, let $\left(\omega_{1} \otimes \omega_{2}, M_{1} \otimes M_{2}\right)$ denote the $\sigma$-continuous representation of $S$ into the $W^{*}$-tensor product $M_{1} \otimes M_{2}$ by

$$
\left(\omega_{1} \otimes \omega_{2}\right)(s)=\omega_{1}(s) \otimes \omega_{2}(s)
$$

for each $s \in S$.

Proposition 5.5. Let $x$ be a nonzero element in $W^{*}(S)$. Then the followings are equivalent:

(a) $x \in \sigma(F(S))$. 
(b) $\quad h_{\omega_{1} \otimes \omega_{2}}(x)=h_{\omega_{1}}(x) \otimes h_{\omega_{2}}(x)$ for any $\sigma$-continuous *-representations $\left(\omega_{i}, M_{i}\right), i=1,2$, of $S$.

(c) $h_{\omega_{\Omega} \otimes \omega_{\Omega}}(x)=x \otimes x$.

Proof. $\quad(\mathrm{a}) \Longrightarrow(\mathrm{b}) . \quad$ Let $\phi_{i} \in\left(M_{i}\right)_{*} . \quad$ Then $\left(\phi_{1} \otimes \phi_{2}\right)^{\wedge}=\hat{\phi}_{1} \cdot \hat{\phi}_{2}$. Hence

$$
\begin{aligned}
\left\langle h_{\omega_{1} \otimes \omega_{2}}(x), \phi_{1} \otimes \phi_{2}\right\rangle & =\left\langle x,\left(\phi_{1} \otimes \phi_{2}\right)^{\wedge}\right\rangle \\
& =\left\langle x, \hat{\phi}_{1} \cdot \hat{\phi}_{2}\right\rangle \\
& =\left\langle x, \hat{\phi}_{1}\right\rangle\left\langle x, \hat{\phi}_{2}\right\rangle \\
& =\left\langle h_{\omega_{1}}(x), \phi_{1}\right\rangle\left\langle h_{\omega_{2}}(x), \phi_{2}\right\rangle \\
& =\left\langle h_{\omega_{1}}(x) \otimes h_{\omega_{2}}(x), \phi_{1} \otimes \dot{\phi}_{2}\right\rangle
\end{aligned}
$$

using Theorem $4.1(\mathrm{~b})$. Since $\left\{\phi_{1} \otimes \phi_{2}, \phi_{i} \in\left(M_{i}\right)_{*}\right\}$ is total in $\left(M_{1} \otimes M_{2}\right)_{*}$, (b) follows.

(b) $\Rightarrow$ (c) is clear.

(c) $\Rightarrow$ (a). Let $f_{1}, f_{2} \in F(S)$ and $\psi_{1}, \psi_{2}$ be the unique elements in $W^{*}(S)_{*}$ such that $\hat{\psi}_{i}=f_{i}$. Then

$$
\begin{aligned}
\left\langle f_{1}, x\right\rangle\left\langle f_{2}, x\right\rangle & =\left\langle\psi_{1}, x\right\rangle\left\langle\psi_{2}, x\right\rangle \\
& =\left\langle\psi_{1} \otimes \psi_{2}, x \otimes x\right\rangle \\
& =\left\langle\psi_{1} \otimes \psi_{2}, h_{\omega_{-2} \otimes \omega_{2}}(x)\right\rangle \\
& =\left\langle\left(\psi_{1} \otimes \psi_{2}\right)^{\wedge}, x\right\rangle \quad \text { (by Theorem 4.1(b)) } \\
& =\left\langle\psi_{1} \cdot \hat{\psi_{2}}, x\right\rangle \\
& =\left\langle f_{1} \cdot f_{2}, x\right\rangle .
\end{aligned}
$$

Since $x \neq 0, x \in \sigma(F(S))$.

REMARK 5.6. (a) Both Propositions 5.4 and 5.5 are due to Martin E. Walter ([18, Theorem 1(ii) and (iii)] and [19, Corollary to Theorem 2]) when $S$ is a locally compact group. Our proof of Proposition 5.4 is completely different from that of Walter. However, using Proposition 5.5 and an argument similar to that in [18, Theorem 1(iii)] we can also obtain a part of Proposition 5.4, i.e., if $x, y \in \sigma(F(S))$, then $x^{*} \in \sigma(F(S))$ and $x \cdot y \in \sigma(F(S)) \cup\{0\}$.

(b) It follows from Proposition 5.5 that $\sigma(F(S))$ with the $\sigma$ topology is a compact topological *-semigroup. Also, $\overline{\omega_{\Omega}(S)^{\sigma}}$ is a *-subsemigroup of $\sigma(F(S)) ; \overline{\omega_{\Omega}(S)^{\sigma}}$ is precisely the largest *-compactification of $S$ as defined in [15, Theorem 5.1].

Proposition 5.7. Let $T={\overline{\omega_{\Omega}(S)^{\sigma}}}$. Then there exists a linear isometry and algebra isomorphism $U$ from $F(T)$ onto $F(S)$ such that $U^{*}$ is a $W^{*}$-isomorphism from $W^{*}(S)$ onto $W^{*}(T)$. 
Proof. Let $\omega_{\Omega}^{\prime}$ denote the *-representation of $T$ into $W^{*}(T)$ and let $\omega^{\prime}(s)=\omega_{\Omega}^{\prime}\left(\omega_{\Omega}(s)\right)$ for all $s \in S$. Then $\left(\omega^{\prime}, W^{*}(T)\right)$ is a $\sigma$-continuous ${ }^{*}$-representation of $S$, and $\left\langle\omega^{\prime}(S)\right\rangle^{-\sigma}=W^{*}(T)$. Let $h=h_{\omega^{\prime}}$ (Theorem 4.1). Then $h$ is onto and

$$
h\left(\omega_{\Omega}(s)\right)=\omega_{\Omega}^{\prime}\left(\omega_{\Omega}(s)\right)
$$

for all $s \in S$. Consequently $h(t)=\omega_{\Omega}^{\prime}(t)$ for all $t \in T$. On the other hand, if $k=h_{\omega_{0}}$ where $\omega_{0}$ is the injection map of $T$ into $W^{*}(S)$, then

$$
k\left(\omega_{\Omega}^{\prime}(t)\right)=t
$$

for all $t \in T$. Hence $k(h(t))=t$ for all $t \in T$. Since $\langle T\rangle^{-\sigma}=W^{*}(S)$, it follows that $k(h(x))=x$ for all $x \in W^{*}(S)$. Consequently $h$ is a $W^{*}$-isomorphism. Define $U\left(\psi^{\wedge}\right)=\left(h^{*} \psi\right)^{\wedge}$ for all $\psi \in W^{*}(T)_{*}$. Then $U(f)(s)=f\left(\omega^{\prime}(s)\right)$ for all $s \in S, f \in F(T)$. Hence $U$ is a linear isometry and algebra homomorphism from $F(T)$ onto $F(S)$, and $U^{*}=h$ is a $W^{*}$-isomorphism from $W^{*}(S)$ onto $W^{*}(T)$.

Martin Walter proved in [18] the following beautiful duality theorem: If the Fourier algebras of two locally compact groups $G_{1}$ and $G_{2}$ are isometric isomorphic, then $G_{1}$ and $G_{2}$ are topologically isomorphic. This result, as pointed out in $[18, \mathrm{p} .18]$ is equivalent to B. E. Johnson's isomorphism theorem for the measure algebras of the locally compact groups when $G_{1}$ and $G_{2}$ are abelian. It is easy to see from Proposition 5.7 that Walter's result is no longer valid when $G_{1}, G_{2}$ are topological *-semigroups. However we shall show in the next theorem that if $S_{1}$ and $S_{2}$ are topological *-semigroups with identity and $F\left(S_{1}\right)$ and $F\left(S_{2}\right)$ are isometric isomorphic, then the compact topological *-semigroups $\sigma\left(F\left(S_{1}\right)\right)$ and $\sigma\left(F\left(S_{2}\right)\right)$ are strongly related.

Let $\sigma_{u}(F(S))$ denote all unitary elements in $\sigma(F(S))$ and let $\sigma_{c}(F(S))$ denote the centre of the semigroup $\sigma(F(S))$, i.e., all $x \in \sigma(F(S))$ such that $x \cdot y=y \cdot x$ for all $y \in \sigma(F(S))$. Then $\sigma_{u}(F(S))$ is a group and $\sigma_{c}(F(S))$ is a closed ${ }^{*}$-subsemigroup of $\sigma(F(S))$.

THEOREM 5.8. Let $S_{1}, S_{2}$ be topological *-semigroups with identity. If the Banach algebras $F\left(S_{1}\right)$ and $F\left(S_{2}\right)$ are isometric isomorphic, then there exists a homeomorphism $\phi$ from $\sigma\left(F\left(S_{1}\right)\right)$ onto $\sigma\left(F\left(S_{2}\right)\right)$ such that

(a) $\phi\left(x^{*}\right)=\phi(x)^{*}$ for all $x \in \sigma\left(F\left(S_{1}\right)\right)$.

(b) For each $x, y \in \sigma\left(F\left(S_{1}\right)\right)$, either $\phi(x \cdot y)=\phi(x) \phi(y)$ or $\phi(x \cdot y)=$ $\dot{\phi}(y) \dot{\phi}(x)$.

(c) $\phi$ is $a^{*}$-isomorphism from $\sigma_{c}\left(F\left(S_{1}\right)\right.$ ) onto $\sigma_{c}\left(F\left(S_{2}\right)\right)$.

(d) $\phi$ is either $a^{*}$-isomorphism or $a^{*}$-anti-isomorphism from $\sigma_{u}\left(F\left(S_{1}\right)\right)$ onto $\sigma_{u}\left(F\left(S_{2}\right)\right)$. 
Furthermore, if for each $x \in \sigma\left(F\left(S_{1}\right)\right)$,

$$
\begin{aligned}
& H_{x}=\left\{y \in \sigma\left(F\left(S_{1}\right)\right) ; \phi(x \cdot y)=\phi(y) \phi(x)\right\} \\
& K_{x}=\left\{y \in \sigma\left(F\left(S_{1}\right)\right) ; \phi(x \cdot y)=\phi(x) \phi(y)\right\}
\end{aligned}
$$

and if

$$
H=\cap\left\{H_{x} ; x \in \sigma_{u}\left(F\left(S_{1}\right)\right)\right\} ; \quad K=\bigcap\left\{K_{x} ; x \in \sigma_{u}\left(F\left(S_{2}\right)\right)\right\}
$$

then

(e) $H_{x}, K_{x}$ are $\sigma$-closed subsemigroups of $\sigma\left(F_{1}(S)\right)$ such that $y \in H_{x}$ (resp. $y \in K_{x}$ ) if and only if $y^{*} \in K_{x^{*}}$ (resp. $y^{*} \in K_{x^{*}}$ ).

(f) $H$ and $K$ are $\sigma$-closed *-subsemigroups of $\sigma\left(F\left(S_{1}\right)\right)$ such that $H \cup K=\sigma\left(F_{1}(S)\right)$.

Proof. We follow an idea Martin Walter in the proof of Theorem 2 in [18]. Let $U$ be the isomorphism from $F\left(S_{2}\right)$ onto $F\left(S_{1}\right)$. Since $S_{1}$ has an identity, it follows that $e_{1}$, the identity of $W^{*}\left(S_{1}\right)$, is in $\sigma\left(F\left(S_{1}\right)\right)$. Hence $u=U^{*}\left(e_{1}\right)$ and $v=u^{*}$ are in $\sigma\left(F\left(S_{2}\right)\right)$ (by Proposition 5.4) and $v_{l}$ is an algebra homomorphism from $F\left(S_{2}\right)$ into $F\left(S_{2}\right)$ (Lemma 5.1) such that $\left\|v_{l}(f)\right\|_{\Omega} \leqq\|v\|\|f\|_{\Omega}=\|f\|_{\Omega}$ for all $f \in F\left(S_{2}\right)$. On the other hand, since $u$ is unitary [13, Lemma 12], it follows that

$$
\left\|v_{l}(f)\right\| \leqq\left\|u_{l}\left(v_{l}(f)\right)\right\|=\left\|(v \cdot u)_{l}(f)\right\|=\|f\|
$$

for each $f \in F\left(S_{2}\right)$ by Lemma 5.1, i.e., $v$ is an isometry. Also if $f \in F\left(S_{2}\right)$, then $v_{l}\left(u_{l}(f)\right)=f$. Hence $v_{l}$ is onto. Consequently $U \circ v_{l}$ is also an isometric isomorphism from $F\left(S_{2}\right)$ onto $F\left(S_{1}\right)$. Let $\Phi=$ $\left(U \circ v_{l}\right)^{*}$. Then $\Phi$ is an isometry from $W^{*}\left(S_{1}\right)$ onto $W^{*}\left(S_{2}\right)$. Also

$$
\left\langle\Phi\left(e_{1}\right), f\right\rangle=\left\langle U^{*}\left(e_{1}\right), v_{l}(f)\right\rangle=\left\langle u, v_{l}(f)\right\rangle=\left\langle e_{2}, f\right\rangle
$$

for all $f \in F\left(S_{1}\right)$, where $e_{2}$ is the identity of $W^{*}\left(S_{2}\right)$, by Lemma 5.1. Hence $\Phi\left(e_{1}\right)=e_{2}$. By Theorem 7 in [13], $\Phi$ is a Jordan *-isomorphism from $W^{*}\left(S_{1}\right)$ onto $W^{*}\left(S_{2}\right)$. Let $\phi$ be the restriction of $\Phi$ to $\sigma\left(F\left(S_{1}\right)\right.$ ). Then clearly $\phi$ is a homomorphism from $\sigma\left(F\left(S_{1}\right)\right)$ onto $\sigma\left(F\left(S_{2}\right)\right)$. We shall show that $\phi$ has all desired properties.

That (a) and (c) hold follow from Theorem 5 and Lemma 8 in [13].

To prove (b), we first note that if $x y=y x$, then (b) holds by [13, Theorem 5]. Otherwise, using [13, Lemma 6], we have

$$
\phi(x) \phi(y)+\phi(y) \phi(x)=\phi(x y)+\phi(y x) .
$$

If $\phi(x y) \neq \phi(y) \phi(x)$ and $\phi(x y) \neq \phi(x) \phi(y)$, then $\phi(x y), \phi(y x), \phi(x) \phi(y)$ and $\phi(y) \phi(x)$ are pairwise distinct elements in $\sigma\left(F\left(S_{2}\right)\right)$. However, elements in $\sigma\left(F\left(S_{2}\right)\right)$ are linearly independent [4, p. 93], which is impossible. Hence (b) holds. 
If (e) holds, then clearly $H$ and $K$ are *-subsemigroups of $\sigma\left(F\left(S_{1}\right)\right)$. Also, if $x \in \sigma\left(F\left(S_{1}\right)\right)$, then $H_{x} \cap \sigma_{u}\left(F\left(S_{1}\right)\right)$ and $K_{x} \cap \sigma_{u}\left(F\left(S_{1}\right)\right)$ are subgroups of $\sigma_{u}\left(F\left(S_{1}\right)\right)$ with union equal to $\sigma_{u}\left(F\left(S_{1}\right)\right)$ by (b). Hence either $\sigma_{u}\left(F\left(S_{1}\right)\right) \subseteq H_{x}$ or $\sigma_{u}\left(F\left(S_{1}\right)\right) \leqq K_{x}$. Hence $H \cup K=\sigma\left(F\left(S_{1}\right)\right)$ and (f) holds. Also a similar argument shows that either $\sigma_{u}\left(F\left(S_{1}\right)\right) \subseteq H$ or $\sigma_{u}\left(F\left(S_{1}\right)\right) \subseteq K$. Hence (d) follows readily from [13, Lemma 12].

It remains to prove (e). By Theorem 10 in [13], there exists a central projections $z_{i} \in W^{*}\left(S_{i}\right)_{*}$ such that $\Phi$ is a ${ }^{*}$-isomorphism from $W^{*}\left(S_{1}\right) z_{1}$ onto $W^{*}\left(S_{2}\right) z_{2}$ and a ${ }^{*}$-anti-isomorphism from $W^{*}\left(S_{1}\right)\left(e_{1}-z_{1}\right)$ onto $W^{*}\left(S_{2}\right)\left(e_{2}-z_{2}\right)$. Then $\Phi\left(x z_{1}\right)=\Phi(x) z_{1}$ and $\Phi\left(x\left(e_{1}-z_{1}\right)\right)=\Phi(x)\left(e_{2}-z_{2}\right)$ for all $x \in W^{*}\left(S_{1}\right)$. Also observe that

$$
y \in H_{x} \quad \text { if and only if }(x y-y x) z_{1}=0
$$

and

$$
y \in K_{x} \quad \text { if and only if }(x y-y x) z_{2}=0 .
$$

To prove (1), let $y \in H_{x}$. Then

$$
\begin{aligned}
\Phi\left((x y-y x) z_{1}\right) & =\Phi(x y-y x) z_{2} \\
& =\Phi(y) \Phi(x) z_{2}-\Phi\left(y z_{1}\right) \Phi\left(x z_{1}\right) \\
& =0 .
\end{aligned}
$$

Hence $(x y-y x) z_{1}=0$. Conversely, if $(x y-y x) z_{1}=0$ and $y \notin K_{x}$, then $y \in H_{x}$ by (b). If $y \in K_{x}$, then $(x y-y x)\left(e_{1}-z_{1}\right)=0$. Hence $x y=y x$. So $y \in H_{x}$ by [13, Theorem 5]. (2) can be proved similarly.

Now if $y_{1}, y_{2} \in H_{x}$, then

$$
x\left(y_{1} y_{2}\right) z_{1}=\left(x y_{1}\right) z_{1} y_{2}=\left(y_{1} x\right) z_{1} y_{2}=y_{1}\left(x y_{2}\right) z_{1}=\left(y_{1} y_{2}\right) x z_{1} \text {. }
$$

Hence $y_{1} y_{2} \in H_{x}$ by (1). Similarly we show that $y \in H_{x}$ if and only if $y^{*} \in H_{x^{*}}$ and that $H_{x}$ is $\sigma$-closed. The assertions on $K_{x}$ can be proved by using (2).

REMARK 5.9. (a) Martin Walter [18, Theorem 1(i)] proved that if $G$ is a locally compact group, then $\sigma_{u}(F(G))$ is topologically isomorphic to $G$.

(b) Let $S$ be a topological *-semigroup and $T=\overline{\omega_{\Omega}(S)}$. It follows from Proposition 5.7 and its proof that there exists a homeomorphism and *-isomorphism $\phi$ from $\sigma(F(S))$ onto $\sigma(F(T))$ such that $\phi\left(\omega_{\Omega}(s)\right)=\omega_{\Omega}^{\prime}\left(\omega_{\Omega}(s)\right)$ for all $s \in S$, where $\omega_{\Omega}$ and $\omega_{2}^{\prime}$ denote the *-representations of $S, T$ into $W^{*}(S)$ and $W^{*}(T)$ respectively.

Proposition 5.10. Let $S_{1}, S_{2}$ be topological *-semigroups with identity. If there exists a Banach algebra isomorphism $U$ from $F\left(S_{2}\right)$ onto $F\left(S_{1}\right)$ such that $U$ maps $P\left(S_{2}\right)$ onto $P\left(S_{1}\right)$ and $\|U f\|_{\infty}=\|f\|_{\infty}$ 
for all $f \in F\left(S_{2}\right)$, then there exists a homeomorphism $\phi$ from $\overline{\omega_{\Omega}\left(S_{1}\right)^{\sigma}}$ onto $\overline{\omega_{\Omega}\left(S_{2}\right)^{\sigma}}$ such that (i) $\phi\left(x^{*}\right)=\phi(x)^{*}$ for all $x \in \overline{\omega_{\Omega}\left(S_{1}\right)^{\sigma}}$ and (ii) for any $x, y \in \overline{\omega_{\Omega}\left(S_{1}\right)}$, either $\phi(x \cdot y)=\phi(x) \cdot \phi(y)$ or $\phi(x \cdot y)=\phi(y) \cdot \phi(x)$.

Proof. The assumption implies that $U^{*}$ takes the identity of $W^{*}\left(S_{1}\right)$ to the identity of $W^{*}\left(S_{2}\right)$. Hence if $\phi$ is the restriction of $U^{*}$ to $\overline{\omega_{\Omega}\left(S_{1}\right)^{\sigma}}$, then it follows from the proof of Theorem 5.8 that $\phi$ has properties (i) and (ii). Also an argument similar to that in [7, p. 99] shows that the $\overline{\omega_{\Omega}\left(S_{i}\right)^{\sigma}}=\left\{x \in \sigma\left(F\left(S_{i}\right)\right) ;\langle f, x\rangle \mid \leqq\|f\|_{\infty}\right.$ for all $\left.f \in F\left(S_{i}\right)\right\}$. Hence $\phi$ is a homeomorphism mapping $\overline{\omega_{\Omega}\left(S_{1}\right)^{\sigma}}$ onto $\overline{\omega_{\Omega}\left(S_{2}\right)^{\sigma}}$.

REMARK 5.11. (a) Theorem 5.8 remains valid when either $S_{1}$ or $S_{2}$ has identity. Do the conclusions of Theorem 5.8 still hold when both $S_{1}$ and $S_{2}$ are assumed not to have an identity?

(b) The following questions are posted to us by the referee: Do the hypotheses of Theorem $5.8 \mathrm{imply}$ anything about a sup-norm isometry between $F\left(S_{1}\right)$ and $F\left(S_{2}\right)$ ? (It is true for groups by Walter's result.) Also can one deduce any relationship between $\overline{\omega_{\Omega}\left(S_{1}\right)^{\circ}}$ and $\overline{\omega_{\Omega}\left(S_{2}\right)^{\sigma}}$ ? (See Proposition 5.10.)

\section{REFERENCES}

1. R. F. Arens, The adjoint of a bilinear operation, Proc. Amer. Math. Soc., 2 (1951), 839-848.

2. W. Arverson, An Invitation to $C^{*}$-algebras, Springer-Verlag, 1976.

3. C. Berg and J. P. R. Christensen, Positive definite functions on abelian semigroups, Math. Ann., 223 (1976), 253-272.

4. F. F. Bonsall and J. Duncan, Complete Normed Algebras, Springer-Verlag, 1973.

5. C. F. Dunkl and D. E. Ramirez, $L^{\infty}$-representations of commutative semitopological semigroups, Semigroup Forum, 7 (1974), 180-199.

6. - Representations of commutative semitopological semigroups, Lecture notes in Mathematics, 435 (1975), Springer-Verlag.

7. - Locally compact subgroups of the spectrum of the measure algebra, Semigroup Forum, 3 (1971), 95-107.

8. J. Ernest, Hopf-von Neuman Algebras, in "Proc. Conference Functional Analysis (Irvine, Calif., 1966)". Academic Press, New York 1967, 195-215.

9. — A new group algebra for locally compact groups I, Amer. J. Math., 86 (1964), 467-492.

10. P. Eymard, L'algèbre de Fourier d'un groupe localement compact, Bull. Soc. Math., France, 92 (1964), 181-236.

11. I. Glicksberg, Weak compactness and separate continuity, Pacific J. Math., 11 (1961), 205-214.

12. E. Hewitt and K. A. Ross, Abstract Harmonic Analysis I, Springer-Verlag, 1963.

13. R. V. Kadison, Isometries of operator algebras, Ann. of Math., 54 (1951), 325-338.

14. A. Lau, Analysis on a class of Banach algebras with applications to harmonic analysis on locally compact groups and semigroups.

15. R. J. Lindahl and P. H. Maserick, Positive definite functions on involution semigroups, Duke Math. J., 38 (1971), 771-782. 
16. S. Sakai, $C^{*}$-algebras and $W^{*}$-algebras, Springer-Verlag, 1971.

17. E. Størmer, On the Jordan structure of $C^{*}$-algebras, Trans. Amer. Math. Soc., 120 (1965), 438-447.

18. M. E. Walter, $W^{*}$-algebras and nonabelian harmonic analysis, J. Functional Analysis, 11 (1972), 17-38.

19. - On the structure of the Fourier-Stieltjes algebra, Pacific J. Math., 58 (1975), 267-281.

Received March 16, 1977 and in revised form December 7, 1977.

UNIVERSITY OF ALBERTA

Edmonton, Alberta, Canada T6G 2G1 



\section{PACIFIC JOURNAL OF MATHEMATICS}

\section{EDITORS}

RICHARD ARENS (Managing Editor)

University of California

Los Angeles, California 90024

C. W. Curtis

University of Oregon

Eugene, OR 97403

C. C. MOORE

University of California

Berkeley, CA 94720

\section{J. DUGUNDJI}

Department of Mathematics University of Southern California Los Angeles, California 90007

R. Finn AND J. Milgram Stanford University Stanford, California 94305

\section{ASSOCIATE EDITORS}

E. F. BeCK ENBACH

B. H. NeUMaNN

F. WOLF

K. Yoshida

\section{SUPPORTING INSTITUTIONS}

UNIVERSITY OF BRITISH COLUMBIA CALIFORNIA INSTITUTE OF TECHNOLOGY UNIVERSITY OF CALIFORNIA MONTANA STATE UNIVERSITY UNIVERSITY OF NEVADA, RENO NEW MEXICO STATE UNIVERSITY OREGON STATE UNIVERSITY UNIVERSITY OF OREGON
UNIVERSITY OF SOUTHERN CALIFORNIA STANFORD UNIVERSITY UNIVERSITY OF HAWAII UNIVERSITY OF TOKYO UNIVERSITY OF UTAH WASHINGTON STATE UNIVERSITY UNIVERSITY OF WASHINGTON 


\section{Pacific Journal of Mathematics \\ Vol. 77, No. $1 \quad$ January, 1978}

Dan Amir, Chebyshev centers and uniform convexity ............... 1

Lawrence Wasson Baggett, Representations of the Mautner group. I ..... 7

George Benke, Trigonometric approximation theory in compact totally

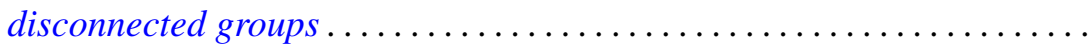

M. Bianchini, O. W. Paques and M. C. Zaine, On the strong compact-ported topology for spaces of holomorphic mappings ..................

Marilyn Breen, Sets with $(d-2)$-dimensional kernels

J. L. Brenner and Allen Kenneth Charnow, Free semigroups of $2 \times 2$

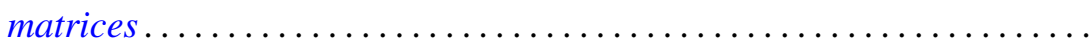

David Bressoud, A new family of partition identities .................

David Fleming Dawson, Summability of matrix transforms of stretchings

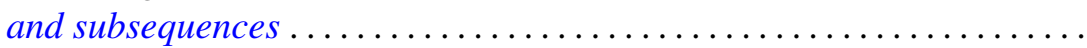

Harold George Diamond and Paul Erdôs, A measure of the nonmonotonicity

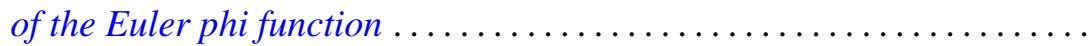

Gary Doyle Faulkner and Ronald Wesley Shonkwiler, Kernel dilation in reproducing kernel Hilbert space and its application to moment

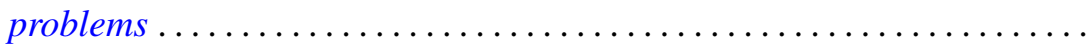

Jan Maksymilian Gronski, Classification of closed sets of attainability in the

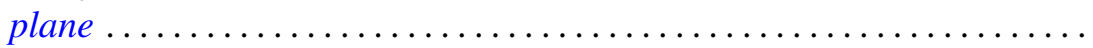

H. B. Hamilton and T. E. Nordahl, Semigroups whose lattice of congruences

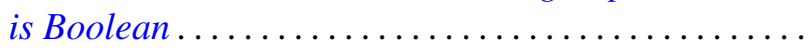

Harvey Bayard Keynes and D. Newton, Minimal $(G, \tau)$-extensions ...

Anthony To-Ming Lau, The Fourier-Stieltjes algebra of a topological

semigroup with involution.

B. C. Oltikar and Luis Ribes, On prosupersolvable groups ...

Brian Lee Peterson, Extensions of pro-affine algebraic groups ...

Thomas M. Phillips, Primitive extensions of Aronszajn spaces ...

Mehdi Radjabalipour, Equivalence of decomposable and 2-decomposable operators. .

M. Satyanarayana, Naturally totally ordered semigroups .

Fred Rex Sinal, A homeomorphism classification of wildly imbedded two-spheres in $S^{3}$

Hugh C. Williams, Some properties of a special set of recurring

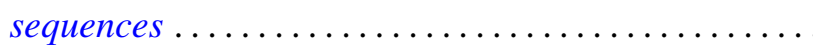

